\title{
Active Brownian ring polymers
}

Cite as: J. Chem. Phys. 150, 064913 (2019); https://doi.org/10.1063/1.5082723

Submitted: 23 November 2018 . Accepted: 15 January 2019 . Published Online: 13 February 2019

S. Mahdiyeh Mousavi (D), Gerhard Gompper (D), and Roland G. Winkler (D)
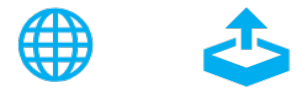


\title{
Active Brownian ring polymers
}

\author{
Cite as: J. Chem. Phys. 150, 064913 (2019); doi: 10.1063/1.5082723 \\ Submitted: 23 November 2018 • Accepted: 15 January 2019 • \\ Published Online: 13 February 2019
}

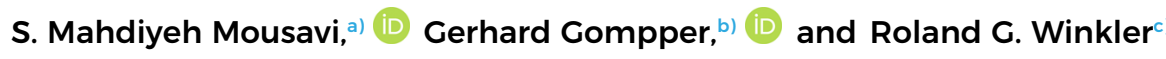

\begin{abstract}
AFFILIATIONS
Theoretical Soft Matter and Biophysics, Institute of Complex Systems and Institute for Advanced Simulation, Forschungszentrum Jülich, D-52425 Jülich, Germany

Note: This article is part of the Special Topic "Chemical Physics of Active Matter" in J. Chem. Phys.

a) Electronic mail: m.mousavi@fz-juelich.de

${ }^{b)}$ Electronic mail: g.gompper@fz-juelich.de

${ }^{c}$ Electronic mail: r.winkler@fz-juelich.de
\end{abstract}

\begin{abstract}
The conformational and dynamical properties of semiflexible active Brownian ring polymers are investigated analytically. A ring is described by the Gaussian semiflexible polymer model accounting for the finite contour length. Activity is implemented by a Gaussian, non-Markovian stochastic process resembling either an external nonthermal force or a local self-propulsion velocity as for an active Ornstein-Uhlenbeck particle. Specifically, the fluctuation spectrum of normal-mode amplitudes is analyzed. At elevated activities, flexible (tension) modes dominate over bending modes even for semiflexible rings, corresponding to enhanced conformational fluctuations. The fluctuation spectrum exhibits a crossover from a quadratic to a quartic dependence on the mode number with increasing mode number, originating from intramolecular tension, but the relaxation behavior is either dominated by intra-polymer processes or the active stochastic process. A further increase in activity enhances fluctuations at large length scales at the expense of reduced fluctuations at small scales. Conformationally, the mean square ring diameter exhibits swelling qualitatively comparable to liner polymers. The ring's diffusive dynamics is enhanced, and the mean square displacement shows distinct activity-determined regimes, consecutively, a ballistic, a subdiffusive, and a diffusive regime. The subdiffusive regime disappears gradually with increasing activity.
\end{abstract}

Published under license by AIP Publishing. https://doi.org/10.1063/1.5082723

\section{INTRODUCTION}

Living matter, or more generally, active matter is inherently out of equilibrium. ${ }^{1-7}$ By converting either internal energy into directed motion or utilizing energy from the environment, active matter agents maintain a nonequilibrium steady state, which is associated with a persistent active stress. $^{8-11}$ Such stresses are particularly interesting since there is no equilibrium counterpart and, thus, they usually cannot be described by equilibrium thermodynamics. Active stresses emerge in ensembles of individual active objects, e.g., in form of swim stress, 8,1 1-13 but also in tightly linked objects such as active polymers ${ }^{14-22}$ or membranes, ${ }^{23-25}$ which are either active themselves or are exposed to an active environment, as internal stress/tension, i.e., bond forces or membrane tension. The latter tensions are associated with nonthermal, active fluctuations. Such fluctuations affect the structural, conformational, and dynamical properties of soft matter systems, e.g., comprised of filaments, polymers, or vesicles/cells, which render active soft matter a promising new class of materials. ${ }^{6}$ The connectivity of active particles, as in linear chains ${ }^{14,15,17,20,21,26-39}$ or other arrangements, e.g., triangles or squares, ${ }^{40}$ gives rise to particular interesting conformational and dynamical features due to the intimate coupling between conformation and activity.

Nature provides a wide range of active agents or phenomena, where activity governs the nonequilibrium dynamics of polymer-like structures. Linear polymers, such as filamentous actin or microtubules of the cell cytoskeleton are propelled by tread-milling and motor proteins. ${ }^{41-43}$ Similarly, in motility assays, filaments are propelled on carpets of motor proteins anchored on a substrate. ${ }^{21,44-52}$ Moreover, the active dynamics of microtubules ${ }^{53}$ or actinfilaments ${ }^{54}$ enhances the dynamics of chromosomal loci ${ }^{55,56}$ 
and chromatin..$^{57-59}$ Here, computer simulations ${ }^{16-18,29}$ and theoretical studies ${ }^{14,15,20-22,37,38,60}$ confirm the decisive influence of activity on the polymer properties.

Less attention has been paid to active cyclic polymer structures, despite their relevance in biological systems. Adenosine triphosphate (ATP)-dependent enzymatic activityinduced mechanical fluctuations drive molecular motion in the cytoplasm of bacteria and eukaryotic cells ${ }^{55}$ and affect the properties of cyclic DNA molecules. ${ }^{61,62}$ In a more abstract sense, in two dimensions, a ring polymer is also a model of a vesicle or cell membrane. ${ }^{63-67}$ As mentioned above, active processes in cells lead to active fluctuations. ${ }^{24,25}$ Hence, studies of active circular polymers shed light onto the properties of a range of biologically relevant systems.

In this article, we analytically analyze the conformational and dynamical characteristics of active semiflexible ring polymers, denoted as active Brownian ring polymers (ABRPs) in the following, including activity-induced shape fluctuations. We describe the ring by the continuous Gaussian semiflexiblepolymer model with the constraint of a unit mean square tangent vector. ${ }^{68-70}$ Activity is taking into account as colored noise, i.e., by a Gaussian but non-Markovian stochastic process. ${ }^{18,20,37-39,60,71}$ Studies of the properties of linear polymers by the same model $20,38,39$ yield substantial conformational changes with varying activity. Flexible linear polymers exhibit a monotonic swelling with increasing activity, which saturates in the asymptotic limit of very large activity. Semiflexible linear polymers shrink for moderate activities ${ }^{16,18,20}$ and swell similar to flexible ones for large activities. ${ }^{20}$ For any stiffness, the intramolecular relaxation times decrease with increasing activity, i.e., the dynamics is accelerated. Moreover, the overall diffusive dynamics is enhanced, an activitytypical ballistic regime appears, and the internal, polymercharacteristic dynamics is modified. In particular, the segmental mean square displacement (MSD) of semiflexible polymers is slowed down and exhibits a time dependence similar to a passive flexible polymer at larger activities. ${ }^{38}$ These findings are in qualitative ${ }^{18,26,72}$ and even in quantitative ${ }^{73}$ agreement with computer simulation results. Ring polymers, specifically flexible rings, exhibit a similar qualitative behavior, although there are quantitative differences mainly due to the distinct boundary conditions and the correspondingly different wave-number spectra.

Active (ring) polymers exhibit enhanced shape fluctuations, which we characterize by the normal-mode amplitude spectrum. As a consequence of independent and additive thermal and active fluctuations, the normal mode spectrum is the sum of passive and active contributions, with activitydependent relaxation times. For flexible and moderately stiff rings, the spectrum of passive rings exhibits a crossover from a tension-dominated to a bending-mode governed regime with increasing mode number. This passive contribution decreases with increasing activity, and, already for moderate activities, the fluctuation spectrum is dominated by activity. Interestingly, here tension modes dominate the fluctuation spectrum over a wide range of length scales, with a crossover from a quadratic to a quartic, bending-mode-type dependence on the mode number with decreasing wavelength. With increasing activity, the fluctuations at larger length scales increase substantially and the spectrum exhibits a bendingmode-type characteristics from large to small length scales. The fluctuation spectrum shows a pronounced dependence on the ring contour length. For large contour lengths, the largelength-scale increase of the mode amplitudes is markedly less pronounced than for smaller contour lengths.

The manuscript is organized as follows: The model, its solution, and the relaxation-time spectrum are presented in Sec. II. In Sec. III, conformational properties, and in Sec. IV, the fluctuation spectrum, are discussed. The dynamical properties are analyzed in Sec. V. Finally, Sec. VI summarizes our findings and results.

\section{MODEL: ACTIVE BROWNIAN RING POLYMER}

\section{A. Equation of motion}

We consider a polymer as a continuous, differentiable space curve $r(s, t)$ of length $L$ embedded in three dimensions, with the contour coordinate $s(0<s<L)$ along the contour of the ring evolving as function of time t. Activity is introduced by assigning the local velocity $\boldsymbol{v}(s, t)$ to the point $\boldsymbol{r}(\mathrm{s}$, $t$ ), which changes in a diffusive manner (cf. Fig. 1). By applying the Gaussian semiflexible polymer model, ${ }^{20,68,69}$ the equation of motion of an ABRP is given by the overdamped Langevin equation $^{38}$

$$
\frac{\partial \boldsymbol{r}(\mathrm{s}, \mathrm{t})}{\partial \mathrm{t}}=\boldsymbol{v}(\mathrm{s}, \mathrm{t})+\frac{1}{\gamma} \boldsymbol{\Gamma}(\mathrm{s}, \mathrm{t})+\frac{k_{\mathrm{B}} \mathrm{T}}{\gamma}\left[2 \boldsymbol{v} \frac{\partial^{2} \boldsymbol{r}(\mathrm{s}, \mathrm{t})}{\partial \mathrm{s}^{2}}-\epsilon \frac{\partial^{4} \boldsymbol{r}(\mathrm{s}, \mathrm{t})}{\partial \mathrm{s}^{4}}\right] .
$$

Activity can be considered either as a result of linked active Brownian particles (ABPs) $)^{1,2,13,20}$ or active OrnsteinUhlenbeck particles (AOUPs) ${ }^{5,74}$ along the ring contour (cf. Fig. 1) or by a nonthermal interaction of a passive ring with its environment. In any case, the propulsion velocity is described by a Gaussian, non-Markovian stochastic process with zero mean and the correlation function ${ }^{20}$

$$
\left\langle\boldsymbol{v}(\mathrm{s}, \mathrm{t}) \cdot \boldsymbol{v}\left(\mathrm{s}^{\prime}, \mathrm{t}^{\prime}\right)\right\rangle=v_{0}^{2} l e^{-\gamma_{\mathrm{R}}\left|\mathrm{t}-\mathrm{t}^{\prime}\right|} \delta\left(\mathrm{s}-\mathrm{s}^{\prime}\right) .
$$

Here, the length $l$ is introduced in the continuum representation of the semiflexible polymer (Fig. 1). In a touching-bead

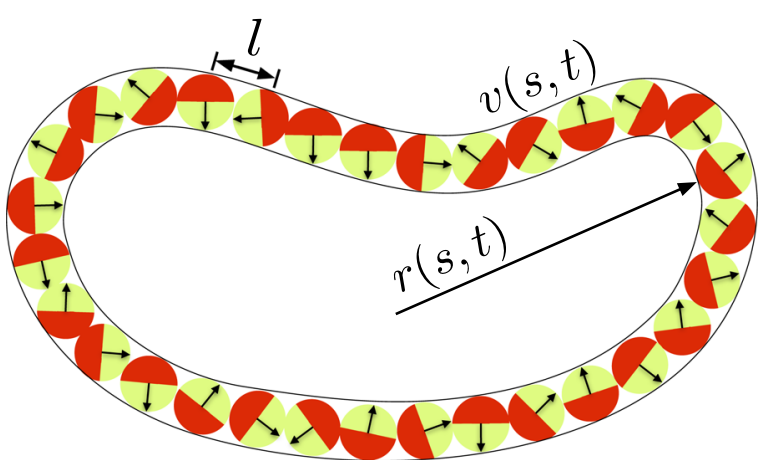

FIG. 1. Illustration of an active Brownian ring polymer. 
model of a discrete polymer, $l$ corresponds to the bead diameter and introduces a minimal length scale. The stationary Gaussian and Markovian stochastic process $\boldsymbol{\Gamma}(\mathrm{s}, \mathrm{t})$ with zero mean and the second moments

$$
\left\langle\Gamma_{\alpha}(\mathrm{s}, \mathrm{t}) \Gamma_{\beta}\left(\mathrm{s}^{\prime}, \mathrm{t}^{\prime}\right)\right\rangle=2 \gamma k_{\mathrm{B}} \mathrm{T} \delta_{\alpha \beta} \delta\left(\mathrm{t}-\mathrm{t}^{\prime}\right) \delta\left(\mathrm{s}-\mathrm{s}^{\prime}\right)
$$

account for thermal fluctuations, $\mathrm{T}$ is the temperature, $k_{\mathrm{B}}$ the Boltzman constant, $\gamma$ the translational friction coefficient per length, and $\alpha, \beta \in\{x, y, z\}$. The term with the fourth-order derivative in Eq. (1) describes intrinsic bending restrictions. Generally, the bending coefficient $\epsilon$ of a three dimensional polymer is solely determined by its persistence length, i.e., $\epsilon=3 /(4 p)$, where $p$ is related to the persistence length $l_{p}$ via $p=1 /\left(2 l_{p}\right){ }^{69}$ The term with the second-order derivative accounts for the conformational entropy of the ring polymer, where the stretching coefficient $v$ is a Lagrangian multiplier related to the inextensibility of the polymer in a mean-field manner. ${ }^{68,69,75,76}$ Inextensibility of its contour is an intrinsic characteristic of a polymer. We capture this important aspect by the local constraint of a unit mean square tangent vector ${ }^{69}$

$$
\left\langle\left(\frac{\partial r(s, t)}{\partial s}\right)^{2}\right\rangle=1 \text {. }
$$

By the ring nature, the polymer properties are translationally invariant along its contour, hence, the constraint and the Lagrangian multiplier $v$ are independent of the contour coordinate s. Omission of this polymer property, as in approximations often used in theoretical analyses of Rouse models of active polymers, leads to wrong results especially at moderate and large activities.

\section{B. Eigenfunction expansion}

The solution of the linear inhomogeneous partial differential equation (1) follows by the expansion

$$
r(s, t)=\sum_{n=-\infty}^{\infty} \chi_{n}(t) \varphi_{n}(s)
$$

in terms of the eigenfunctions $\varphi_{n}(\mathrm{~s})$ of the equation

$$
\epsilon k_{\mathrm{B}} \mathrm{T} \frac{d^{4}}{d \mathrm{~s}^{4}} \varphi_{n}(\mathrm{~s})-2 v k_{\mathrm{B}} \mathrm{T} \frac{d^{2}}{d \mathrm{~s}^{2}} \varphi_{n}(\mathrm{~s})=\xi_{n} \varphi_{n}(\mathrm{~s}) .
$$

The periodic boundary condition $\boldsymbol{r}(s, t)=\boldsymbol{r}(s+L, t)$ for a ring yields

$$
\varphi_{n}(\mathrm{~s})=\frac{1}{\sqrt{\mathrm{L}}} e^{i \zeta_{n} \mathrm{~s}}, \quad \varphi_{0}=\frac{1}{\sqrt{\mathrm{L}}},
$$

with the wave numbers $\zeta_{n}=2 n \pi / L, n \in \mathbb{Z} \backslash 0$, and the eigenvalues $\xi_{n}=k_{\mathrm{B}} \mathrm{T}\left(\epsilon \zeta_{n}^{4}+2 v \zeta_{n}^{2}\right)$. The wave numbers are independent of activity, in contrast to linear polymers, which somewhat simplifies the analytical treatment. ${ }^{38}$ Yet, the eigenvalues, $\xi_{n}$, depend on activity via the stretching coefficient $v$. Inserting the eigenfunction expansion (5) into Eq. (1) yields the equation of motion for the mode amplitudes $\chi_{n}(t)$

$$
\begin{aligned}
& \frac{d}{d t} \chi_{n}(t)=-\frac{1}{\tau_{n}} \chi_{n}(t)+\frac{1}{\gamma} \boldsymbol{\Gamma}_{n}(t)+\boldsymbol{v}_{n}(t), \quad n \neq 0, \\
& \frac{d}{d t} \chi_{0}(t)=\frac{1}{\gamma} \boldsymbol{\Gamma}_{0}(t)+\boldsymbol{v}_{(0)}(t),
\end{aligned}
$$

with the relaxation times ${ }^{77}$

$$
\tau_{n}=\frac{\gamma}{\xi_{n}}=\frac{\gamma}{k_{\mathrm{B}} \mathrm{T}\left(\epsilon \zeta_{n}^{4}+2 v \zeta_{n}^{2}\right)} .
$$

In analogy to $\boldsymbol{\chi}_{n}(\mathrm{t}), \boldsymbol{v}_{n}(\mathrm{t})$ and $\boldsymbol{\Gamma}_{n}(\mathrm{t})$ are the normal-mode amplitudes of the active velocity and the stochastic force, respectively. The stationary-state solution of Eq. (8) and the solution of Eq. (9) are

$$
\begin{aligned}
& \chi_{n}(t)=\int_{-\infty}^{t} e^{-\left(t-t^{\prime}\right) / \tau_{n}}\left[\boldsymbol{v}_{n}\left(t^{\prime}\right)+\boldsymbol{\Gamma}_{n}\left(t^{\prime}\right)\right] d t^{\prime}, \\
& \chi_{0}(t)=\chi_{0}(0)+\int_{0}^{t}\left[\boldsymbol{v}_{(0)}\left(t^{\prime}\right)+\boldsymbol{\Gamma}_{0}\left(t^{\prime}\right)\right] d t^{\prime} .
\end{aligned}
$$

\section{Correlation functions}

The correlation functions of the amplitudes $\boldsymbol{\Gamma}_{n}(t)$ and $\boldsymbol{v}_{n}(t)$ of the thermal and active noise are given by

$$
\begin{aligned}
\left\langle\boldsymbol{v}_{n}(\mathrm{t}) \cdot \boldsymbol{v}_{m}\left(\mathrm{t}^{\prime}\right)\right\rangle & =v_{0}^{2} l e^{-\gamma_{\mathrm{R}}\left|t-\mathrm{t}^{\prime}\right|} \delta_{m n}, \\
\left\langle\boldsymbol{\Gamma}_{n}(\mathrm{t}) \cdot \boldsymbol{\Gamma}_{m}\left(\mathrm{t}^{\prime}\right)\right\rangle & =6 \gamma k_{\mathrm{B}} \mathrm{T} \delta\left(t-\mathrm{t}^{\prime}\right) \delta_{m n} .
\end{aligned}
$$

With them, we obtain for the correlations of the mode amplitudes $\chi_{n}$

$$
\begin{aligned}
\left\langle\chi_{n}(t) \cdot \chi_{m}\left(t^{\prime}\right)\right\rangle= & \delta_{n m}\left(\frac{3 k_{\mathrm{B}} \mathrm{T} \tau_{n}}{\gamma} e^{-\left|t-t^{\prime}\right| / \tau_{n}}\right. \\
& \left.+\frac{v_{0}^{2} l \tau_{n}^{2}}{1-\left(\gamma_{\mathrm{R}} \tau_{n}\right)^{2}}\left[e^{-\gamma_{\mathrm{R}}\left|t-\mathrm{t}^{\prime}\right|}-\gamma_{\mathrm{R}} \tau_{n} e^{-\left|t-\mathrm{t}^{\prime}\right| / \tau_{n}}\right]\right),
\end{aligned}
$$

as basis for further calculations of second-order correlations and moments.

\section{CONFORMATIONAL PROPERTIES}

\section{A. Lagrangian multiplier: Stretching coefficient}

In terms of the eigenfunction expansion (5) and the modecorrelation functions (15), the constraint (4) becomes

$$
\sum_{n=-\infty}^{\infty}\left(\frac{3 k_{\mathrm{B}} \mathrm{T}}{\gamma} \tau_{n}+\frac{v_{0}^{2} l}{1+\gamma_{\mathrm{R}} \tau_{n}} \tau_{n}^{2}\right)\left|\frac{d \varphi_{n}(\mathrm{~s})}{d s}\right|^{2}=1 .
$$

We introduce dimensionless quantities, by defining the Péclet number Pe, characterizing activity, and the ratio $\Delta$ between the translation, $D_{T}=k_{B} T /(\gamma l)$, and rotational, $D_{R}$ $=2 \gamma_{R}$ (in three dimensions), diffusion coefficient as

$$
\mathrm{Pe}=\frac{v_{0}}{\mathrm{D}_{\mathrm{R}} l}, \quad \Delta=\frac{\mathrm{D}_{\mathrm{T}}}{\mathrm{D}_{\mathrm{R}} l^{2}}
$$

in analogy to an ABP/AOUP. ${ }^{74}$ This choice is motivated by a bead-spring polymer model as used in simulations of active polymers, ${ }^{17,18,73}$ where $l$ corresponds to the bead diameter (bond length). In the following, we set $\Delta=1 / 3$, the value of a passive colloid in solution. Then, we obtain the nonlinear relation 


$$
\sum_{n=1}^{\infty}\left(\frac{1}{\hat{\xi}_{n}}+\frac{P e^{2}}{3 \Delta\left(3 \Delta l^{3} \hat{\xi}_{n}^{2}+2 \hat{\xi}_{n}\right)}\right) \zeta_{n}^{2}=\frac{L}{2}
$$

to determine the stretching coefficient $v$. Here, the abbreviation

$$
\hat{\xi}_{n}=\frac{\xi_{n}}{3 k_{\mathrm{B}} \mathrm{T}}=\frac{4 \pi^{2} p \mu}{\mathrm{L}^{2}}\left(n^{2}+\frac{\pi^{2} n^{4}}{\mu(p L)^{2}}\right)
$$

and the scaled stretching coefficient $\mu=2 v /(3 p)$ are introduced. The sum over the mode numbers in Eq. (18) can be evaluated analytical, as shown in the supplementary material.

Depending on persistence length and activity, the following asymptotic behavior for the stretching coefficient $\mu$ is obtained.

\section{Passive ring polymer}

Without activity, Pe = 0, Eq. (18) yields

$$
\frac{1}{\sqrt{\mu}} \operatorname{coth}(\sqrt{\mu} p L)-\frac{1}{\mu p L}=1 \text {. }
$$

In the limit of a flexible ring, $p L \gg 1, \operatorname{coth}(\sqrt{\mu} p L) \rightarrow 1$, and the stretching coefficient becomes $\mu=1$. However, for semiflexible rings, $p L \lesssim \pi, \mu$ is negative (cf. Fig. 2), which leads to

$$
\frac{1}{|\mu| p L}-\frac{1}{\sqrt{\mu}} \cot (\sqrt{|\mu|} p L)=1 .
$$

Here, Taylor expansion for $p L \ll 1$ yields

$$
\mu=-\frac{\pi^{2}}{(p L)^{2}}\left(1-\frac{2 p L}{\pi^{2}}\right)
$$

The negative internal tension opposes thermal fluctuations, which attempt to shrink and crumple the circular structure, and maintains the (near) circular structure.

Figure 2(a) displays the stretching coefficient $\mu$ as function of $\mathrm{Pe}$ for various $p \mathrm{~L}$, which corresponds to different persistence lengths $l_{p}=1 /(2 p)$ at fixed ring-contour length The values at $\mathrm{Pe} \rightarrow 0$ reflect the result for passive rings.

\section{Active ring polymer}

Activity implies an increase of the stretching coefficient with increasing Pe (cf. Fig. 2); $\mu$ assumes only positive values for $p L \gtrsim 3\left(N=10^{2}, \Delta=1 / 3\right)$. Hence, the internal tension grows with activity. Analytical approximations for the asymptotic behavior of long polymers or large activity can be obtained.

In the limit $p L \gg 1$, Eq. (18) turns into

$$
\begin{aligned}
& \frac{1}{\sqrt{\mu}} \operatorname{coth}(\sqrt{\mu} p L)-\frac{1}{\mu p L}-\frac{\mathrm{Pe}^{2}}{6 \mu p L \Delta} \\
& +\frac{\mathrm{Pe}^{2}}{(6 \mu p L \Delta)^{3 / 2}} \operatorname{coth}\left(\sqrt{\frac{\mathrm{N}^{3}}{6 p L \mu \Delta}}\right)=1
\end{aligned}
$$

because the contribution of the bending modes $\left(\sim n^{4}\right)$ of Eq. (19) can be neglected and the sum is dominated by the entropic modes $\left(\sim n^{2}\right)$.

The solution of Eq. (23) depends on the ration $L / l=N$, i.e., on the polymer length. This is a consequence of our definition of the Péclet number with the length scale $l$, but allows for a straightforward comparison with simulations of discrete active bead-spring models. For a flexible polymer, we set $\mathrm{L} / \mathrm{l}$ $=p L$. Hence, the argument $x \equiv \sqrt{\mathrm{N}^{3} / 6 p L \mu \Delta}$ of $\operatorname{coth}(x)$ is proportional to $p L / \sqrt{\mu}$, and $\operatorname{coth}(x) \rightarrow 1$ for $p L / \sqrt{\mu} \gg 1$. Thus, we obtain the Péclet-number dependence

$$
\mu=\frac{\mathrm{Pe}^{4 / 3}}{6 \Delta}
$$

in the limit $p \mathrm{~L} \rightarrow \infty$ and $1 \ll \mathrm{Pe}<\infty$.

In contrast, in case of $1 \ll p L<\infty$ and $\mathrm{Pe} \rightarrow \infty$, i.e., $\boldsymbol{\mu} \rightarrow \infty$, the argument of $\operatorname{coth}(x)$ is small, and the Taylor expansion yields

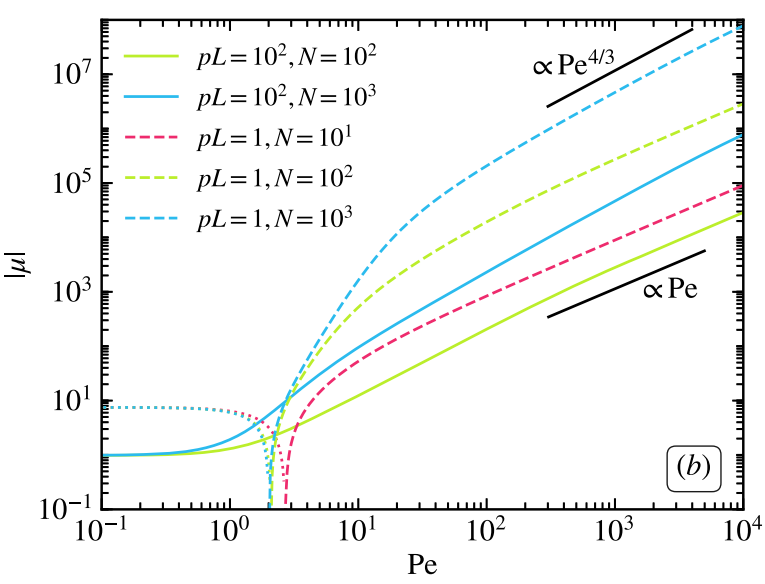

FIG. 2. Modulus of the stretching coefficient $\mu=2 v /(3 p)$ as a function of Péclet number. (a) Variation of $\mu$ with the bending stiffness (pL) for $L / I=N=10^{2}$. (b) Variation of $\mu$ with $N$ for $p L=10^{2}$ (solid lines) and $p L=1$ (dashed lines). The dotted lines indicate the negative part of $\mu$ for the respective $p L$, which appears for $p L \leqslant \pi$. With increasing $\mathrm{Pe}, \mu$ crosses zero, which leads to a rapid change of $|\mu|$. The black solid lines indicate power-law dependencies. 


$$
\mu=\frac{\mathrm{PeN}^{3 / 2}}{6 \sqrt{3} p \mathrm{~L} \Delta} .
$$

These results are, aside from a constant factor, equal to the dependencies determined for a linear flexible active polymer. ${ }^{20}$

A similar crossover in the power-law dependence appears for semiflexible polymers, $p \mathrm{~L} \lesssim 1$, and large Péclet numbers. For $\mu \gg \pi^{2} /(p L)^{2}$, which is achieved for large enough Pe, the eigenvalues $\hat{\xi}_{n}$ are dominated by $n^{2}$ modes, and $\mu$ follows from Eq. (23) too. Depending on $\mathrm{N}, \boldsymbol{\mu}$ is given by Eq. (24) or (25). Hence, Eq. (25) describes the asymptotic limit Pe $\rightarrow \infty$, or $\mu \rightarrow \infty$, for any stiffness.

Figure 2(b) illustrates the dependence of the stretching coefficient on the ring contour length for two $p L$ values, i.e., stiffness. The two distinct slopes are clearly visible. Every curve exhibits a slope of unity in the limit Pe $\rightarrow \infty$, but significantly larger $\mu$ values are assumed for stiffer polymers $(\mathrm{Pe} \gtrsim 3)$.

The crossover from negative (compression) to positive (stretching) tension shifts to larger Péclet numbers with decreasing $p L$. When the active contribution to the ring dynamics dominates, shape fluctuations are large and tension crosses over to positive values. Here, activity is strong enough to crumple and shrink the ring. For Pe smaller than the value at the zero-crossing of $\mu$, the conformational properties are determined by thermal fluctuations.

\section{B. Relaxation times}

The constraint of a unit mean square tangent vector results in a dependence of the relaxation times

$$
\tau_{n}=\frac{\gamma \mathrm{L}^{2}}{12 \pi^{2} k_{\mathrm{B}} \mathrm{Tp}} \frac{1}{\mu}\left(\frac{1}{n^{2}+\pi^{2} n^{4} /\left[\mu(p L)^{2}\right]}\right)
$$

on the activity via the stretching coefficient, $\mu$, which gives rise to a distinct mode-number dependence of $\tau_{n}$ for flexible and semiflexible ABRPs. In the limits $p L \gg 1$ or Pe $\gg 1(\mu \gg 1)$, the relaxation times reduce to

$$
\tau_{n}=\frac{\gamma \mathrm{L}^{2}}{12 \pi^{2} k_{\mathrm{B}} \mathrm{Tp}} \frac{1}{\mu n^{2}} .
$$

Hence, the relaxation times exhibit the same mode-number dependence as a linear flexible polymer. Since $\mu$ increases monotonically for sufficiently large Pe (cf. Fig. 2), the relaxation times decrease with increasing Pe, i.e., activity accelerates relaxation processes.

Bending modes can dominate the relaxation times for semiflexible rings, $p L \lesssim 1$. In the asymptotic limit $p L \rightarrow 0$, the longest relaxation time [Eq. (26)] of a passive ring is

$$
\tau_{1}=\frac{\gamma \mathrm{L}^{3}}{24 \pi^{2} k_{\mathrm{B}} \mathrm{T}}
$$

which corresponds to its average rotational relaxation time. Note that $\boldsymbol{\mu}$, Eq. (22), is negative in this regime. This relaxation time is by a factor of $2 \pi^{2} / 3$ smaller than that of a comparable linear polymer. ${ }^{20}$ Figure $3(\mathrm{a})$ depicts the longest relaxation time $\tau_{1}$ as a function of Péclet number for various persistence lengths. For $\mathrm{Pe} \rightarrow 0$ and $p \mathrm{~L} \rightarrow 0$, the predicted value of Eq. (28) is assumed. With increasing $p L(p L \gtrsim 10), \tau_{1}$ decrease as $\tau_{1} / L^{3}$ $\sim 1 /(p L)$ as expected for a flexible (linear, ring) polymer. An increasing Péclet number, $\mathrm{Pe}>1$, leads to a decrease of the relaxation time due to the monotonic increase of $\mu$. Yet, the drop of the relaxation times for a semiflexible ring is significantly stronger in the regime $1<\mathrm{Pe} \lesssim 10^{2}$ due to the stronger dependence of $\boldsymbol{\mu}$ on Pe [cf. Fig. 2(b)]. Since $\tau_{1} \sim 1 / \mu$ asymptotically [Eq. (27)], the power laws of Eqs. (24) and (25) are obtained in the corresponding limit, i.e., the dependencies $\tau_{1} \sim 1 / \mathrm{Pe}^{4 / 3}$ and $\tau_{1} \sim 1 / \mathrm{Pe}$, where the latter applies for $\mathrm{Pe} \rightarrow \infty$.

The dependence of the relaxation time on the mode number for a rather stiff ring polymer is presented in Fig. 3(b). As expected, passive stiff rings exhibit a bendingmode-dominated behavior with $\tau_{n} \sim 1 / n^{4}$. With increasing
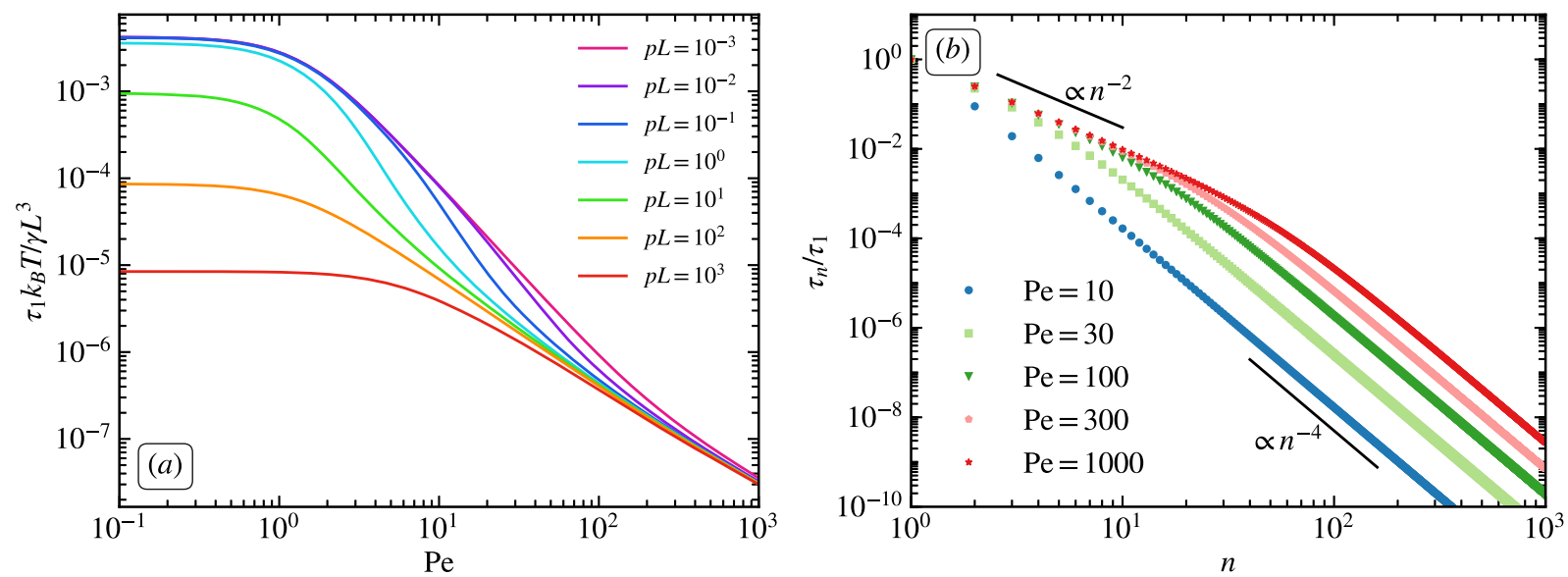

FIG. 3. (a) Longest relaxation time $\tau_{1}$ as a function of Péclet number for various bending stiffness from flexible $\left(p L=10^{3}\right)$ to stiff $\left(p L=10^{-3}\right)$. (b) Dependence of the relaxation times $\tau_{n}$ on the mode number for various Péclet numbers for $p L=10^{-1}$. The $\tau_{n}$ are normalized by the longest relaxation time $\tau_{1}$. In (a) and (b), the ring contour length is $L / l$ $=N=10^{2}$. 
activity, flexible modes, $1 / n^{2}$, prevail at small mode numbers, and bending modes for $n>\sqrt{\mu} p L / \pi$. The crossover between the two regimes shifts to larger mode numbers with increasing activity. This indicates that semiflexibility matters at large mode numbers, i.e., at small length scales.

As is well known, passive flexible linear polymers exhibit a $1 / n^{2}$ drop of the relaxation times at small mode numbers, and a crossover to bending modes for $n>p L / \pi .{ }^{77}$

\section{Ring diameter and radius of gyration}

We characterize the conformations of the ring by the mean square ring diameter $\left\langle\boldsymbol{r}_{d}^{2}\right\rangle=\left\langle(\boldsymbol{r}(\mathrm{L} / 2)-\boldsymbol{r}(0))^{2}\right\rangle$, which is given by

$$
\left\langle\boldsymbol{r}_{d}^{2}\right\rangle=\frac{8}{\mathrm{~L}} \sum_{n=1}^{\infty}\left\langle\chi_{2 n-1}^{2}\right\rangle
$$

in terms of the mean square mode amplitudes

$$
\left\langle\chi_{n}^{2}\right\rangle=\frac{3 k_{\mathrm{B}} \mathrm{T}}{\gamma} \tau_{n}+\frac{v_{0}^{2} l}{1+\gamma_{\mathrm{R}} \tau_{n}} \tau_{n}^{2}
$$

The sum over modes for any mean square distance $\langle(\boldsymbol{r}(\mathrm{s})-$ $\left.\left.r\left(s^{\prime}\right)\right)^{2}\right\rangle$ can be evaluated analytically, as shown in the supplementary material. Similarly, the radius of gyration

$$
\left\langle\boldsymbol{r}_{g}^{2}\right\rangle=\frac{1}{2 \mathrm{~L}^{2}} \int_{0}^{\mathrm{L}} \int_{0}^{\mathrm{L}}\left\langle\left(\boldsymbol{r}(\mathrm{s})-\boldsymbol{r}\left(\mathrm{s}^{\prime}\right)\right)^{2}\right\rangle d s d \mathrm{~s}^{\prime}=\frac{2}{\mathrm{~L}} \sum_{n=1}^{\infty}\left\langle\boldsymbol{\chi}_{n}^{2}\right\rangle
$$

characterizes the ring structure.

Numerical results for the mean square diameter are presented in Fig. 4. For flexible rings, $\left\langle\boldsymbol{r}_{d}^{2}\right\rangle$ increases monotonically with increasing Péclet number and saturates in the limit $\mathrm{Pe} \rightarrow \infty$ at a $p L$-independent value. Semiflexible rings $(p L$ $\lesssim 10$ ) shrink at moderate Pe, pass through a minimum and swell again with increasing Pe, where the swelling behavior is rather similar. Hence, flexible and semiflexible ring polymers exhibit

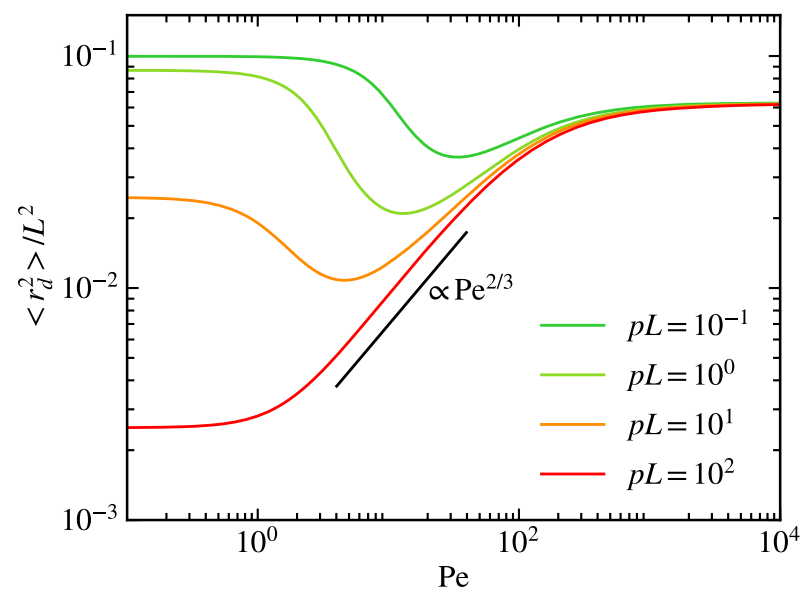

FIG. 4. Mean square diameter $\left\langle\boldsymbol{r}_{d}^{2}\right\rangle=\left\langle(\boldsymbol{r}(\mathrm{L} / 2)-\boldsymbol{r}(0))^{2}\right\rangle$ of ABRPs as a function of Péclet number $\mathrm{Pe}$ for various persistence lengths. The contour length is $L / I$ $=10^{2}$. The black solid line indicates the predicted power-law regime of Eq. (37). a distinctly different active response at moderate Péclet numbers, but show similar conformations at large Pe due to the dominance of flexible modes at sufficiently large activities (cf. Sec. IV for a discussion of fluctuations). The actual shrinkage and swelling depends on the contour length $\mathrm{N}=\mathrm{L} / l$, where shrinkage of semiflexible rings is more pronounced for larger $\mathrm{N}$. The asymptotic dependencies on the ring length, persistence length, and Péclet number can be obtained analytically.

\section{Passive ring polymer}

Evaluation of the sum in Eqs. (29) and (31) for a passive ring yields

$$
\left\langle\boldsymbol{r}_{d}^{2}\right\rangle / \mathrm{L}^{2}=\frac{1}{4 p \mathrm{~L} \mu}-\frac{1}{2(p L)^{2} \mu^{3 / 2}} \tanh \left(\frac{p \mathrm{~L}}{2} \sqrt{\mu}\right),
$$

$$
\left\langle\boldsymbol{r}_{g}^{2}\right\rangle / \mathrm{L}^{2}=\frac{1}{4 \mu p \mathrm{~L}}\left(\frac{1}{3}+\frac{1}{(p L)^{2} \boldsymbol{\mu}}-\frac{1}{p L \sqrt{\mu}} \operatorname{coth}(p L \sqrt{\mu})\right) .
$$

Asymptotically, we obtain in the limit of flexible rings, $p L \gg 1$,

$$
\left\langle\boldsymbol{r}_{d}^{2}\right\rangle=\frac{L}{4 p}, \quad\left\langle\boldsymbol{r}_{g}^{2}\right\rangle=\frac{L}{12 p}=\frac{\left\langle\boldsymbol{r}_{d}^{2}\right\rangle}{3} .
$$

The mean square diameter is identical with the mean square end-to-end distances of a flexible linear polymer of length $\mathrm{L} / 2$. Hence, flexible linear and ring polymers exhibit the same conformational properties on comparable length scales.

In the opposite limit of stiff rings, $p L \rightarrow 0$, we find

$$
\left\langle\boldsymbol{r}_{d}^{2}\right\rangle=\frac{\mathrm{L}^{2}}{\pi^{2}}, \quad\left\langle\boldsymbol{r}_{g}^{2}\right\rangle=\frac{\mathrm{L}^{2}}{4 \pi^{2}} .
$$

Both quantities reflect the circular shape of the polymer, namely, the circumference $L=\pi \sqrt{\left\langle r_{d}^{2}\right\rangle}$, and the moment of inertia in terms of the circle radius.

\section{Active ring polymer}

The general expression for the mean square ring diameter follows from the general mean square distance result provided in the supplementary material for $s=0$ and $s^{\prime}=L / 2$. Here, we analyze properties for long polymers, $p L \gg 1$, and/or large Péclet numbers, Pe $\gg 1$, in more detail. Then, the relaxation times (26) are dominated by flexible modes (entropy) as long as $n \ll \sqrt{\mu} p L / \pi$, and we obtain

$$
\begin{aligned}
\left\langle r_{d}^{2}\right\rangle / \mathrm{L}^{2}= & \frac{1}{4 p \mathrm{~L} \mu}-\frac{1}{2(p \mathrm{~L})^{2} \mu^{3 / 2}} \tanh \left(\frac{p \mathrm{~L}}{2} \sqrt{\mu}\right) \\
& +\mathrm{Pe}^{2}\left[\frac{1}{24 \mu p \mathrm{~L} \Delta}-\frac{1}{2 \sqrt{6 \mu p L N^{3} \Delta}} \tanh \left(\frac{\sqrt{\mathrm{N}^{3}}}{2 \sqrt{6 \mu p \mathrm{~L} \Delta}}\right)\right]
\end{aligned}
$$

The choice $N=L / l=p L(p L \rightarrow \infty)$ and $1 \ll \operatorname{Pe}<\infty$ then yields

$$
\left\langle r_{d}^{2}\right\rangle=\frac{\mathrm{L}^{2} \mathrm{Pe}^{2 / 3}}{4 p \mathrm{~L}}
$$


with $\boldsymbol{\mu}$ of Eq. (24). Hence, the mean square ring diameter increases in a power-law fashion over a large rang of Péclet numbers for long and flexible polymers. This is in agreement with the full numerical solution displayed Fig. 4. The mean square end-to-end distance of linear polymers exhibits the same power-law dependence. ${ }^{20}$

In the asymptotic limit $\mathrm{Pe} \rightarrow \infty$ and $p \mathrm{~L}<\infty$, we find

$$
\left\langle\boldsymbol{r}_{d}^{2}\right\rangle=\frac{L^{2}}{16}
$$

independent of stiffness, again in agreement with the full solution.

The mean square radius of gyration exhibits qualitatively the same behavior as $\left\langle\boldsymbol{r}_{d}^{2}\right\rangle$. Thus, we will not discuss it in detail, except for its asymptotic behavior

$$
\left\langle\boldsymbol{r}_{g}^{2}\right\rangle= \begin{cases}\frac{\mathrm{L}^{2} \mathrm{Pe}^{2 / 3}}{12 p \mathrm{~L}}=\frac{\left\langle\boldsymbol{r}_{d}^{2}\right\rangle}{3}, & 1 \ll \mathrm{Pe}<\infty ; p \mathrm{~L} \rightarrow \infty, \\ \frac{\mathrm{L}^{2}}{60}=\frac{16\left\langle\boldsymbol{r}_{d}^{2}\right\rangle}{60}, & \mathrm{Pe} \rightarrow \infty .\end{cases}
$$

Therefore, for flexible rings $(p L \rightarrow \infty)$, the same ratio is obtained for passive and active rings. Even the ratio $\left\langle\boldsymbol{r}_{g}^{2}\right\rangle /\left\langle\boldsymbol{r}_{d}^{2}\right\rangle$ is close to the passive value in the limit $\mathrm{Pe} \rightarrow \infty$.

Activity causes swelling of rings, similar to linear polymers. Yet, even in the asymptotic limit of large Péclet numbers, no perfect circular shape is assumed. The active fluctuations rather result in distorted structures with large fluctuations.

We like to emphasize that theories on swelling of Rousetype active polymers, which ignore the constraint on the tangent vector, predict a quadratic dependence of $\left\langle\boldsymbol{r}_{d}^{2}\right\rangle$ and $\left\langle\boldsymbol{r}_{g}^{2}\right\rangle$ on the Peclet number up to infinitely large Pe, and no saturation of the deformation occurs. Hence, such models predict a different Péclet-number dependence than that derived by our approach even at moderate Pe. Note that our analytical results for linear polymers nearly quantitatively agree with simulation results of $A B P$ polymers, ${ }^{73}$ confirming the derived dependence on Pe and the saturation.

\section{NONEQUILIBRIUM SHAPE FLUCTUATIONS}

The shape fluctuations are characterized by the mean square mode amplitudes of Eq. (30) in the stationary state. The fluctuations are nonlinear functions of relaxation times and Péclet number. The linear equation of motion of the polymer, with independent stochastic processes for the translational motion (thermal noise) and active velocity, yields two additive contributions to $\left\langle\chi_{n}^{2}\right\rangle,\left\langle\chi_{n}^{2}\right\rangle_{\text {th }}$ and $\left\langle\chi_{n}^{2}\right\rangle_{a}$. The amplitudes

$$
\left\langle\chi_{n}^{2}\right\rangle_{\text {th }}=\frac{3 k_{\mathrm{B}} \mathrm{T} \tau_{n}}{\gamma}=\frac{\mathrm{L}^{3}}{4 \pi^{2} p L \mu n^{2}\left(1+\pi^{2} n^{2} /\left[\mu(p L)^{2}\right]\right)}
$$

originate from thermal fluctuations. In the absence of activity $(\mu=1)$, Eq. (40) formally reflects equipartition of energy, although we have to keep in mind that the flexible contribution represents the conformational freedom of the ring (entropy). However, in the nonequilibrium state, the relaxation times are functions of activity via the stretching parameter $\mu$, i.e., the tension in the ring, which increases with increasing activity. The active forces $\gamma \boldsymbol{v}(s, t)$ amplify fluctuations. This is particularly evident for a semiflexible ring $(p L$ $<1)$, where the active forces enhance preferentially transverse fluctuations and additionally imply small length scale undulations, i.e., flexible modes. The same applies to flexible polymers, where $\left\langle\chi_{n}^{2}\right\rangle_{\text {th }} / \mathrm{L}^{3}=1 /\left(4 \pi^{2} p L \mu n^{2}\right) \sim 1 /(\mu p L) \sim l_{p} /(\mu \mathrm{L})$. Hence, $\mu$ reduces the persistence length $\left(l_{p} / \mu\right)$ and, thus, the larger length scale fluctuations. As a consequence, the fluctuations $\left\langle\chi_{n}^{2}\right\rangle_{\text {th }}$ decrease for all modes with increasing activity (cf. Fig. 3) to an extent that they are negligible compared to the active fluctuations

$$
\left\langle\chi_{n}^{2}\right\rangle_{a}=\frac{v_{0}^{2} l}{1+\gamma_{\mathrm{R}} \tau_{n}} \tau_{n}^{2}=\frac{\mathrm{Pe}^{2} l^{3}}{4} \frac{\gamma_{\mathrm{R}}^{2} \tau_{n}^{2}}{1+\gamma_{\mathrm{R}} \tau_{n}},
$$

where $\tau_{n}^{-1} \sim n^{2}\left(1+\pi^{2} n^{2} /\left[\mu(p L)^{2}\right]\right)$. Figure 5 illustrates the decreasing fluctuations $\left\langle\chi_{n}^{2}\right\rangle_{\text {th }}$ with increasing Pe, and $\left\langle\chi_{n}^{2}\right\rangle_{\text {th }}$ is significantly smaller than $\left\langle\chi_{n}^{2}\right\rangle_{a}$ over an increasing range of mode numbers. Only at large mode numbers, the thermal contribution to the fluctuations dominates since it decreases slower $\left(1 / n^{2}\right)$ than the active contribution $\left(1 / n^{4}\right)$ (see the discussion below).

The active fluctuations (41) are determined by two competing factors as Pe increases, the increasing Péclet number itself and the decreasing relaxation times $\tau_{n}$. The quadratic dependence on Pe originates from the dependence of the mode-amplitude fluctuations on the mean square correlation function of the active noise [cf. Eqs. (11) and (12)]. Figure 5 depicts the variation of the fluctuations with the Péclet number. Since the thermal contribution decreases with increasing $\mathrm{Pe},\left\langle\chi_{n}^{2}\right\rangle$ is solely determined by the active contribution (41) for sufficiently large Pe. The fluctuations at small wave numbers $(n \lesssim 10)$ increase with increasing Pe substantially compared to the passive case due to the $\mathrm{Pe}^{2}$ dependence. In addition,

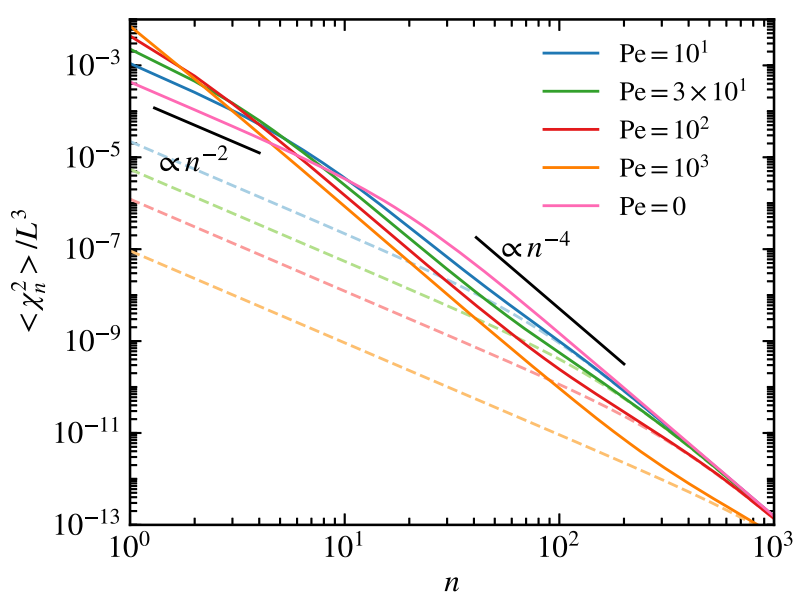

FIG. 5. Mode-amplitude fluctuations as a function of the mode number for various Péclet numbers. The contour length is $L / I=10^{2}$ and $p L=60$. The dashed lines depict contributions by thermal fluctuations (40). 
the mode-number dependence changes from a $1 / n^{2}$ to a $1 / n^{4}$ dependence. However, this is not caused by a dominance of bending modes over flexible modes, but is rather a genuine effect of activity. For Pe $\lesssim 50$ and mode numbers $n<\sqrt{\mu} p L / \pi$, $\gamma_{\mathrm{R}} \tau_{n}>1$, and we can approximate Eq. (41) by

$$
\left\langle\chi_{n}^{2}\right\rangle_{a}=\frac{\mathrm{Pe}^{2} l^{3}}{4} \gamma_{\mathrm{R}} \tau_{n}
$$

Hence, the $\tau_{n}$ of Eq. (27) imply $\left\langle\chi_{n}^{2}\right\rangle_{a} \sim 1 / n^{2}$ for small $n$. Since $\tau_{n}$ decreases with increasing mode number $-\gamma_{R} \tau_{n} \ll 1$ above a certain $n$ or at large Péclet numbers-we find

$$
\left\langle\chi_{n}^{2}\right\rangle_{a}=\frac{\mathrm{Pe}^{2} l^{3}}{4} \gamma_{\mathrm{R}}^{2} \tau_{n}^{2}
$$

and $\left\langle\chi_{n}^{2}\right\rangle_{a} \sim 1 / n^{4}$ for $\tau_{n}$ of Eq. (27), a mode-number dependence characteristic for bending modes. This relation applies down to rather small mode numbers. Yet, the fluctuation spectrum is determined solely by active fluctuations and tension with $\tau_{n} \sim 1 / n^{2}$. Above a Pe-dependent mode number, active fluctuations are smaller than passive ones, e.g., for $\mathrm{Pe}=10$ in the regime $10 \lesssim n \lesssim 10^{2}$ (cf. Fig. 5). Hence, small-scale fluctuations are reduced at the expense of enhanced large-scale fluctuations.

Qualitatively, our fluctuation spectrum agrees with that of fluctuating membranes, ${ }^{24}$ where also an increase of the fluctuations at small wave vectors by activity compared to an equilibrium system has been observed experimentally ${ }^{78}$ and described theoretically. ${ }^{24}$ As for our approach, active membrane fluctuations are smaller than those of a passive system above a certain wave number. ${ }^{24}$ A quantitative comparison with membrane calculations is only meaningful in two dimensions, but our approach has to be augmented by an area constraint to describe vesicles. ${ }^{65}$

\section{DYNAMICS}

The mean square displacement (MSD) $\left\langle\Delta \boldsymbol{r}^{2}(\mathrm{t})\right\rangle=\langle(\boldsymbol{r}(\mathrm{s}, \mathrm{t})-$ $\left.\boldsymbol{r}(\mathrm{s}, 0))^{2}\right\rangle$ of any point $\boldsymbol{r}(\mathrm{s}, \mathrm{t})$ is given by

$$
\left\langle\Delta \boldsymbol{r}^{2}(t)\right\rangle=\left\langle\Delta \boldsymbol{r}_{c m}^{2}(t)\right\rangle+\left\langle\Delta \boldsymbol{r}_{0}^{2}(t)\right\rangle+\left\langle\Delta \boldsymbol{r}_{a}^{2}(\mathrm{t})\right\rangle
$$

independent of its location along the ring contour, with the center-of-mass MSD

$$
\left\langle\Delta \boldsymbol{r}_{\mathrm{cm}}^{2}(t)\right\rangle=\frac{6 k_{\mathrm{B}} \mathrm{T}}{\gamma \mathrm{L}} \mathrm{t}+\frac{2 v_{0}^{2} l}{\gamma_{\mathrm{R}}^{2} \mathrm{~L}}\left(\gamma_{\mathrm{R}} t-1+e^{-\gamma_{\mathrm{R}} t}\right),
$$

the activity-modified equilibrium internal-dynamics contribution

$$
\left\langle\Delta \boldsymbol{r}_{0}^{2}(t)\right\rangle=\sum_{n=1}^{\infty} \frac{12 k_{\mathrm{B}} \mathrm{T} \tau_{n}}{\gamma \mathrm{L}}\left(1-e^{-t / \tau_{n}}\right),
$$

and the active contribution

$$
\left\langle\Delta \boldsymbol{r}_{a}^{2}(t)\right\rangle=\sum_{n=1}^{\infty} \frac{4 v_{0}^{2} l \tau_{n}^{2}}{\mathrm{~L}\left(1+\gamma_{\mathrm{R}} \tau_{n}\right)}\left(1-\frac{e^{-\gamma_{\mathrm{R}} t}-\gamma_{\mathrm{R}} \tau_{n} e^{-t / \tau_{n}}}{1-\gamma_{\mathrm{R}} \tau_{n}}\right) .
$$

The center-of-mass MSD is identical with that of a linear polymer, ${ }^{20,38}$ where activity yields a ballistic motion $\left\langle\Delta \boldsymbol{r}_{\mathrm{cm}}^{2}(\mathrm{t})\right\rangle$ $=v_{0}^{2} l t^{2} / \mathrm{L}$ for $\gamma_{\mathrm{R}} t \ll 1$, and diffusive behavior $\left\langle\Delta \boldsymbol{r}_{\mathrm{cm}}^{2}(t)\right\rangle$ $=2 v_{0}^{2} l t /\left(\gamma_{R} L\right)$ for $\gamma_{R} t \gg 1$. Notably, the center-of-mass MSD strongly depends on the contour length $L / l=N$ since $\left\langle\Delta \boldsymbol{r}_{\mathrm{cm}}^{2}(\mathrm{t})\right\rangle / \mathrm{L}^{2} \sim 1 / \mathrm{N}^{3}$. The $\left\langle\Delta \boldsymbol{r}_{\mathrm{cm}}^{2}(\mathrm{t})\right\rangle$ is identical with the MSD of an active Brownian particle aside from the factor $1 / \mathrm{L}$. For the passive contribution, the factor $\gamma \mathrm{L}$ simply represents the total friction coefficient. For the active contribution to the MSD, the factor $\mathrm{N}$ of the independent AOUPs, with Gaussian distributed velocities, accounts for the excess of monomers pointing in a particular direction.

The active contribution $\left\langle\Delta r_{a}^{2}(t)\right\rangle$ is determined by two independent relaxation processes-the decay of the active velocity characterized by $\gamma_{R}$, and the internal polymer dynamics with the relaxation times $\tau_{n}$.

Figure 6 depicts MSDs for flexible $\left[(a), p L=10^{2}\right]$ and semiflexible $\left[(b), p L=10^{-1}\right]$ rings for various Péclet numbers. Four time regimes for flexible, five for semiflexible, polymers can be identified in the presence of activity, determined by different processes:

- $t \rightarrow \infty$-The MSD (44) is given by the linear center-ofmass diffusion with the diffusion coefficient

$$
\mathrm{D}=\frac{k_{\mathrm{B}} \mathrm{T}}{\gamma \mathrm{L}}+\frac{v_{0}^{2} l}{3 \gamma_{\mathrm{R}} \mathrm{L}}
$$

Equations (46) and (47) together approach the stationary-state value $2\left\langle\boldsymbol{r}_{g}^{2}\right\rangle$.

- $\mathrm{t} / \tau_{1} \ll 1 \ll \gamma_{\mathrm{R}} \mathrm{t}-$ For Pe $\gg 1$, the active contribution is dominated by $\left(\gamma_{\mathrm{R}} \tau_{1} \gg 1\right)$

$$
\left\langle\Delta \boldsymbol{r}_{a}^{2}(t)\right\rangle=\frac{4 v_{0}^{2} l}{\gamma_{\mathrm{R}} L} \sum_{n=1}^{\infty} \tau_{n}\left(1-e^{-t / \tau_{n}}\right) .
$$

With the relaxation times of flexible polymers ( $p L \gg 1)$, Eq. (27), and by replacing the sum by an integral, we obtain

$$
\left\langle\Delta r_{a}^{2}(\mathrm{t})\right\rangle \approx \frac{2 v_{0}^{2} l}{\gamma_{\mathrm{R}} \mathrm{L}} \sqrt{\frac{\tau_{\mathrm{R}}}{\mu}} \mathrm{I}_{2} \sqrt{\mathrm{t}}
$$

where $\tau_{R}=\gamma \mathrm{L}^{2} /\left(3 \pi^{2} k_{\mathrm{B}} \mathrm{T} p\right)$ is the Rouse relaxation time $\mathrm{e}^{79}$ and $\mathrm{I}_{\mathrm{n}}=\int_{0}^{\infty}\left(1-e^{-x^{n}}\right) / x^{n} d x$. Even in the presence of activity, there is a flexible-polymer-specific subdiffusive time regime. The predicted regime is clearly visible in Fig. 6(a) for $\mathrm{Pe}=10$ and, to a less extent, for $\mathrm{Pe}=30$. The regime decreases with increasing activity because $\tau_{1}$ decreases with increasing Pe.

In case of semiflexible polymers, $p L \ll 1$, Eq. (26) yields $\tau_{n}=\tau_{R}(p L)^{2} /\left(4 \pi^{2} n^{4}\right)(n>1)$, and the integral approximation gives

$$
\left\langle\Delta r_{a}^{2}(t)\right\rangle \approx \frac{4 v_{0}^{2} l}{\gamma_{\mathrm{R}} L}\left(\tau_{1}\left(1-e^{-t / \tau_{1}}\right)+\sqrt[4]{\frac{\tau_{\mathrm{R}}(p L)^{2}}{64 \pi^{2}}} \mathrm{I}_{4} \mathrm{t}^{3 / 4}\right)
$$

The first term on the right-hand side describes the average rotational motion of the ring with the relaxation time (28) as long as $\mathrm{Pe} \lesssim 5$. This is reflected in Fig. $6(\mathrm{~b})$ for $t / \tau_{1} \approx 1$. Here, the rotational motion dominates the internal dynamics 

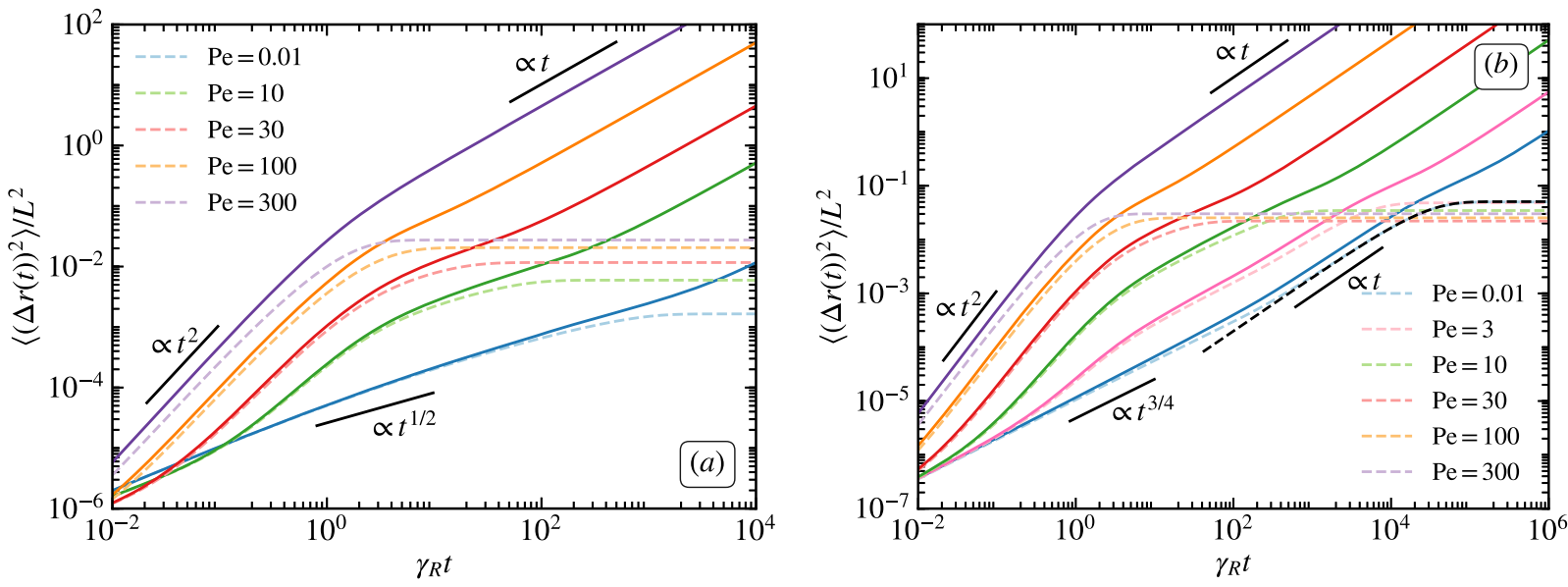

FIG. 6. Mean square displacement of ABRPs with (a) $p L=10^{2}$ and (b) $p L=10^{-1}$ for various Péclet numbers. In both cases, $L / I=10^{2}$. The solid lines present the total MSD [Eq. (44)], whereas the dashed lines refer to the MSD in the center-of-mass reference frame [Eq. (46) plus Eq. (47)].

because contributions from higher order modes are small and decrease linearly with decreasing $p L$. For large Pe, $\tau_{1}$ follows from Eq. (27) and decreases with increasing Pe. In addition, Eq. (51) shows a subdiffusive time regime, now typical for passive semiflexible polymers. ${ }^{80}$ This is visible in Fig. 6(b) for 0 $<$ Pe $\lesssim 5$. As in Fig. 6(a), the regime shortens with increasing activity and decreasing $\tau_{1}$.

- $0 \ll t / \tau_{1}, \gamma_{\mathrm{R}} \mathrm{t} \ll 1$-Taylor expansion of the exponential functions in Eq. (47) yields

$$
\left\langle\Delta \boldsymbol{r}_{a}^{2}(t)\right\rangle=\frac{2 v_{0}^{2} l \gamma_{\mathrm{R}}}{\mathrm{L}} \sum_{n=1}^{\infty} \frac{\tau_{n}}{1+\gamma_{\mathrm{R}} \tau_{n}} \mathrm{t}^{2},
$$

in agreement with the ballistic regimes of Fig. 6.

- $0 \ll t / \tau_{1} \ll 1-$ The thermal contribution (46) yields for $p L \gg 1$

$$
\left\langle\Delta r_{0}^{2}(\mathrm{t})\right\rangle=\frac{6 k_{\mathrm{B}} \mathrm{T}}{\gamma \mathrm{L}} \sqrt{\frac{\tau_{\mathrm{R}} \mathrm{t}}{\mu}} \mathrm{I}_{2},
$$

and for $p L \ll 1$

$$
\left\langle\Delta r_{0}^{2}(t)\right\rangle=\frac{6 k_{\mathrm{B}} \mathrm{T}}{\gamma \mathrm{L}}\left(\tau_{1}\left(1-e^{\mathrm{t} / \tau_{1}}\right)+2 \sqrt[4]{\frac{\tau_{\mathrm{R}}(p \mathrm{~L})^{2}}{4 \pi^{2}}} \mathrm{I}_{4} \mathrm{t}^{3 / 4}\right)
$$

in analogy to Eqs. (50) and (51). The latter is displayed in Fig. 6(b).

- $t \rightarrow 0,0<p L$, Pe $<\infty$-Independent of stiffness and activity, the dynamics is dominated by the relaxation times $\tau_{n}=\tau_{R}(p L)^{2} /\left(4 \pi^{2} n^{4}\right)$. Hence, the mean square displacement is given by the second term of Eq. (54) since this term dominates over the active contribution (52) in the limit $t \rightarrow 0$.

\section{SUMMARY AND CONCLUSIONS}

We have analyzed the conformational and dynamical properties of active Brownian ring polymers, emphasizing the influence of active processes on the normal-mode fluctuations. The ring is described as a Gaussian semiflexible polymer, taking its inextensibility into account in a mean-field manner by the constraint of a unit mean square tangent vector. ${ }^{68,69}$ Activity is modeled as a colored noise force with an exponential temporal correlation. The linearity of the intramolecular forces allows for an analytical solution of the equations of motion (Ornstein-Uhlenbeck process). ${ }^{74,81}$

In general, rings exhibit similar features as linear polymers. Flexible rings monotonically swell with increasing activity, whereas semiflexible rings shrink at moderate activities and swell again at large Péclet numbers. In the asymptotic limit of large Pe, rings swell similarly and assume the same ring-specific finite size, independent of stiffness, however, not a circular shape. Their dynamics is enhanced, with a ballistic activity-determined time regime. However, for moderate Péclet numbers $\mathrm{Pe} \lesssim 50$, a regime governed by the intramolecular polymer dynamics is present, with a power-law MSD characteristic for flexible polymers, $p L \gg 1$ (Rouse dynamics), or for semiflexible polymers, $p L \ll 1$. The later regimes gradually disappear with increasing Pe since the relaxation times simultaneously decrease. The latter is a consequence of the inextensibility of the ring's contour length.

Yet, there are several quantitative and qualitative differences between linear and ring polymers. On the one hand, the wave-number spectrum is distinctly different. The periodic boundary conditions of rings imply activity- and stiffnessindependent wave numbers, in contrast to linear polymers, with strongly Pe-dependent $\zeta_{n} .{ }^{38}$ This affects the relaxationtime spectrum. On the other hand, the intramolecular tension, $\mu$, of rings becomes negative below a certain stiffness ( $p L \lesssim 3$ for passive rings). This regime extends to larger Pe with decreasing $p L$. Active and thermal fluctuations attempt to shrink and crumple a ring-like structure, which is opposed by a negative internal tension. Qualitatively, conformational and dynamical properties of ring and linear polymers at large Péclet numbers are similar, with comparable scaling relations 
for the $p L$ and Pe dependencies, as a consequence of the dominance of the tension modes.

The fluctuation spectrum is dominated by activity already for moderate Péclet numbers, $\mathrm{Pe} \gtrsim 10$ for $\mathrm{N}=10^{2}$, and thermal fluctuations matter only for large mode numbers, i.e., at very small scales. Notably, the major part of the spectrum is determined by tension, with a crossover from a $1 / n^{2}$ to a $1 / n^{4}$ decrease with increasing mode number $n$. Hence, conclusions on underlying fluctuation mechanisms have to be drawn with care, and a $1 / n^{4}$ dependence is not necessarily a sign of dominating bending modes. For the considered rings, the bending-mode-type spectrum appears for $\gamma_{\mathrm{R}} \tau_{n} \ll$ 1 , which is fulfilled for sufficiently large $n$. Here, the dynamics is dominated by the slow relaxation of the active process compared to the fast relaxation of the polymer degrees of freedom.

Our findings on fluctuations are in qualitative agreement with experiments on membranes ${ }^{78}$ and analytical calculations based on the Helfrich free energy extended by active membrane fluctuations. ${ }^{24}$ The latter calculations, comparable with ours, show a similar increase of large-length-scale fluctuations at the expense of reduced small-scale fluctuations. A ring polymer can be considered as a model for a living membrane or a vesicle in two dimensions. A quantitative comparison with two-dimensional closed structures requires, however, a suitable adjustment of the bending and stretching parameters, $\epsilon, v{ }^{80}$

Our consideration of linear and ring polymers, also in comparison with other, analogous models for membranes, reveal various generic features of activity and active fluctuations. Activity dominates the conformational and dynamical properties for $\mathrm{Pe} \gg \sqrt{6 l^{2} \mathrm{D}_{\mathrm{R}} / \mathrm{D}_{\mathrm{T}}}$. Large-scale fluctuations increase at the expense of small-scale fluctuations. However, dynamical quantities, in particular, for $t / \tau_{1} \ll 1$, involve a wide spectrum of modes; hence, active-modulations of fluctuations are hardly visible and discernible, let alone their quantitative characterization via conformational or dynamical quantities.

An interesting aspect is the shrinkage of semiflexible rings by active noise, which should result in an enhanced packing in dense melts of rings and might be important for DNA organization within the cell nucleus. ${ }^{82-84}$ The actual mechanism of DNA packing is unresolved so far; however, DNA transcription or other enzymatic processes, e.g., active-loop extrusion, ${ }^{84,85}$ provide a continuous local energy influx and, hence, a source of nonthermal active noise. A melt of rings as a model system to analyze chromosome territories has explicitly been considered in Ref. 84. Here, extensions accounting for nonequilibrium processes will enhance the understanding on possible mechanisms for DNA packing. In this context, we would like to mention the influence of hydrodynamic interactions on the conformational properties of linear active polymers, which predicted a substantial shrinkage due to hydrodynamic interactions. ${ }^{86}$ Against this background, we will study collective effects in suspension of active polymers and mixtures with passive polymers to resolve the influence of athermal processes on their structural and dynamical properties in the future.
Experimentally, the impact of active noise on polymer properties can be studied by immersing either biological microswimmers, ${ }^{87,88}$ such as bacteria, e.g., E. coli, or synthetic microswimmer ${ }^{89,90}$ in a polymer solution. Typically, the modifications of the microswimmer dynamical properties by the polymer solution have been studied, but not the reverse effect on the polymer characteristics. However, theoretical studies of active polymers exposed to shear flow predict an activity-depending shear thinning. ${ }^{37,39}$ The latter could also play a role in swimming of bacteria. ${ }^{87}$ Dedicated experiments to characterize the polymer properties are feasible and interesting and would yield direct evidence for activity-induced features.

\section{SUPPLEMENTARY MATERIAL}

See supplementary material for analytical expressions for the equation of the stretching coefficient [Eq. (16)] as well as a general expression for the mean square distance between two points on the ring contour.

\section{ACKNOWLEDGMENTS}

We thank T. Auth and C. Abaurrea Velasco (Jülich) for stimulating discussions about active fluctuations of membranes, which have initiated parts of this study. Financial support by the Deutsche Forschungsgemeinschaft (DFG) within the priority Program No. SPP 1726 "Microswimmers-from Single Particle Motion to Collective Behaviour" is gratefully acknowledged.

\section{REFERENCES}

${ }^{1}$ J. Elgeti, R. G. Winkler, and G. Gompper, "Physics of microswimmersSingle particle motion and collective behavior: A review," Rep. Prog. Phys. 78, 056601 (2015).

${ }^{2}$ C. Bechinger, R. Di Leonardo, H. Löwen, C. Reichhardt, G. Volpe, and G. Volpe, "Active particles in complex and crowded environments," Rev. Mod. Phys. 88, 045006 (2016).

${ }^{3}$ A. Bernheim-Groswasser, N. S. Gov, S. A. Safran, and S. Tzlil, "Living matter: Mesoscopic active materials," Adv. Mater. 30, 1707028 (2018).

${ }^{4}$ S. Ramaswamy, “Active matter," J. Stat. Mech.: Theory Exp. 2017, 054002.

${ }^{5}$ É. Fodor, C. Nardini, M. E. Cates, J. Tailleur, P. Visco, and F. van Wijland, "How far from equilibrium is active matter?," Phys. Rev. Lett. 117, 038103 (2016).

${ }^{6}$ M. E. Cates and F. C. MacKintosh, "Active soft matter," Soft Matter 7, 3050 (2011).

${ }^{7}$ É. Fodor and M. C. Marchetti, "The statistical physics of active matter: From self-catalytic colloids to living cells," Physica A 504, 106 (2018).

${ }^{8}$ S. C. Takatori, W. Yan, and J. F. Brady, "Swim pressure: Stress generation in active matter," Phys. Rev. Lett. 113, 028103 (2014).

${ }^{9}$ X. Yang, M. L. Manning, and M. C. Marchetti, "Aggregation and segregation of confined active particles," Soft Matter 10, 6477 (2014).

${ }^{10}$ A. P. Solon, J. Stenhammar, R. Wittkowski, M. Kardar, Y. Kafri, M. E. Cates, and J. Tailleur, "Pressure and phase equilibria in interacting active Brownian spheres," Phys. Rev. Lett. 114, 198301 (2015).

${ }^{11}$ S. Das, G. Gompper, and R. G. Winkler, "Local stress and pressure in an inhomogeneous system of spherical active Brownian particles," Sci. Rep. (submitted).

${ }^{12}$ R. G. Winkler, A. Wysocki, and G. Gompper, "Virial pressure in systems of spherical active Brownian particles," Soft Matter 11, 6680 (2015). 
${ }^{13}$ M. C. Marchetti, Y. Fily, S. Henkes, A. Patch, and D. Yllanes, "Minimal model of active colloids highlights the role of mechanical interactions in controlling the emergent behavior of active matter," Curr. Opin. Colloid Interface Sci. 21, 34 (2016).

${ }^{14}$ T. B. Liverpool, A. C. Maggs, and A. Ajdari, "Viscoelasticity of solutions of motile polymers," Phys. Rev. Lett. 86, 4171 (2001).

${ }^{15}$ A. Ghosh and N. S. Gov, "Dynamics of active semiflexible polymers," Biophys. J. 107, 1065 (2014).

${ }^{16} \mathrm{~J}$. Harder, C. Valeriani, and A. Cacciuto, "Activity-induced collapse and reexpansion of rigid polymers," Phys. Rev. E 90, 062312 (2014).

${ }^{17}$ A. Kaiser and H. Löwen, "Unusual swelling of a polymer in a bacterial bath," J. Chem. Phys. 141, 044903 (2014).

${ }^{18}$ J. Shin, A. G. Cherstvy, W. K. Kim, and R. Metzler, "Facilitation of polymer looping and giant polymer diffusivity in crowded solutions of active particles," New J. Phys. 17, 113008 (2015).

${ }^{19}$ A. Laskar and R. Adhikari, "Brownian microhydrodynamics of active filaments," Soft Matter 11, 9073 (2015).

${ }^{20}$ T. Eisenstecken, G. Gompper, and R. G. Winkler, "Conformational properties of active semiflexible polymers," Polymers 8, 304 (2016).

${ }^{21}$ R. G. Winkler, J. Elgeti, and G. Gompper, "Active polymers-Emergent conformational and dynamical properties: A brief review," J. Phys. Soc. Jpn. 86, 101014 (2017).

${ }^{22}$ H. Löwen, "Active colloidal molecules," Europhys. Lett. 121, 58001 (2018).

${ }^{23}$ S. Tuvia, A. Almagor, A. Bitler, S. Levin, R. Korenstein, and S. Yedgar, "Cell membrane fluctuations are regulated by medium macroviscosity: Evidence for a metabolic driving force," Proc. Natl. Acad. Sci. U. S. A. 94, 5045 (1997).

${ }^{24}$ B. Loubet, U. Seifert, and M. A. Lomholt, "Effective tension and fluctuations in active membranes," Phys. Rev. E 85, 031913 (2012).

${ }^{25}$ H. Turlier, D. A. Fedosov, B. Audoly, T. Auth, N. S. Gov, C. Sykes, J. F. Joanny, G. Gompper, and T. Betz, "Equilibrium physics breakdown reveals the active nature of red blood cell flickering," Nat. Phys. 12, 513 (2016).

${ }^{26}$ D. Sarkar, S. Thakur, Y.-G. Tao, and R. Kapral, "Ring closure dynamics for a chemically active polymer," Soft Matter 10, 9577 (2014).

${ }^{27}$ R. Chelakkot, A. Gopinath, L. Mahadevan, and M. F. , "Flagellar dynamics of a connected chain of active, polar, Brownian particles," J. R. Soc., Interface 11, 20130884 (2014)

${ }^{28}$ D. Loi, S. Mossa, and L. F. Cugliandolo, "Non-conservative forces and effective temperatures in active polymers," Soft Matter 7, 10193 (2011).

${ }^{29}$ R. E. Isele-Holder, J. Elgeti, and G. Gompper, "Self-propelled worm-like filaments: Spontaneous spiral formation, structure, and dynamics," Soft Matter 11, 7181 (2015).

${ }^{30}$ R. E. Isele-Holder, J. Jager, G. Saggiorato, J. Elgeti, and G. Gompper, "Dynamics of self-propelled filaments pushing a load," Soft Matter 12, 8495 (2016).

${ }^{31}$ A. Laskar, R. Singh, S. Ghose, G. Jayaraman, P. B. S. Kumar, and R. Adhikari, "Hydrodynamic instabilities provide a generic route to spontaneous biomimetic oscillations in chemomechanically active filaments," Sci. Rep. 3, 1964 (2013).

${ }^{32}$ G. Jayaraman, S. Ramachandran, S. Ghose, A. Laskar, M. S. Bhamla, P. B. S. Kumar, and R. Adhikari, "Autonomous motility of active filaments due to spontaneous flow-symmetry breaking," Phys. Rev. Lett. 109, 158302 (2012).

${ }^{33} \mathrm{H}$. Jiang and Z. Hou, "Motion transition of active filaments: Rotation without hydrodynamic interactions," Soft Matter 10, 1012 (2014).

${ }^{34}$ S. Babel, H. Löwen, and A. M. Menzel, "Dynamics of a linear magnetic 'microswimmer molecule'," Europhys. Lett. 113, 58003 (2016).

${ }^{35}$ C. Valeriani, M. Li, J. Novosel, J. Arlt, and D. Marenduzzo, "Colloids in a bacterial bath: Simulations and experiments," Soft Matter 7, 5228 (2011).

${ }^{36}$ L. F. Cugliandolo, G. Gonnella, and A. Suma, "Rotational and translational diffusion in an interacting active dumbbell system," Phys. Rev. E 91, 062124 (2015).

${ }^{37}$ R. G. Winkler, "Dynamics of flexible active Brownian dumbbells in the absence and the presence of shear flow," Soft Matter 12, 3737 (2016).
${ }^{38}$ T. Eisenstecken, G. Gompper, and R. G. Winkler, "Internal dynamics of semiflexible polymers with active noise," J. Chem. Phys. 146, 154903 (2017).

${ }^{39}$ A. Martín-Gómez, G. Gompper, and R. G. Winkler, "Active Brownian filamentous polymers under shear flow," Polymers 10, 837 (2018).

${ }^{40}$ N. Küchler, H. Löwen, and A. M. Menzel, "Getting drowned in a swirl: Deformable bead-spring model microswimmers in external flow fields," Phys. Rev. E 93, 022610 (2016).

${ }^{41}$ A. J. Ridley, M. A. Schwartz, K. Burridge, R. A. Firtel, M. H. Ginsberg, G. Borisy, J. T. Parsons, and A. R. Horwitz, "Cell migration: Integrating signals from front to back," Science 302, 1704 (2003).

${ }^{42}$ S. Ganguly, L. S. Williams, I. M. Palacios, and R. E. Goldstein, "Cytoplasmic streaming in Drosophila oocytes varies with kinesin activity and correlates with the microtubule cytoskeleton architecture," Proc. Natl. Acad. Sci. U. S. A. 109, 15109 (2012).

${ }^{43}$ A. Ravichandran, G. A. Vliegenthart, G. Saggiorato, T. Auth, and G. Gompper, "Enhanced dynamics of confined cytoskeletal filaments driven by asymmetric motors," Biophys. J. 113, 1121 (2017).

${ }^{44}$ Y. Harada, A. Noguchi, A. Kishino, and T. Yanagida, "Sliding movement of single actin filaments on one-headed myosin filaments," Nature 326, 805 (1987).

${ }^{45}$ F. J. Nédélec, T. Surrey, A. C. Maggs, and S. Leibler, "Self-organization of microtubules and motors," Nature 389, 305 (1997).

${ }^{46}$ V. Schaller, C. Weber, C. Semmrich, E. Frey, and A. R. Bausch, "Polar patterns of driven filaments," Nature 467, 73 (2010).

${ }^{47}$ F. Jülicher, K. Kruse, J. Prost, and J.-F. Joanny, "Active behavior of the cytoskeleton," Phys. Rep. 449, 3 (2007).

${ }^{48}$ M. C. Marchetti, J. F. Joanny, S. Ramaswamy, T. B. Liverpool, J. Prost, M. Rao, and R. A. Simha, "Hydrodynamics of soft active matter," Rev. Mod. Phys. 85, 1143 (2013).

${ }^{49}$ J. Prost, F. Jülicher, and J.-F. Joanny, "Active gel physics," Nat. Phys. 11, 111 (2015).

${ }^{50}$ A. Cordoba, J. D. Schieber, and T. Indei, "A single-chain model for active gels. I: Active dumbbell model," RSC Adv. 4, 17935 (2014).

${ }^{51}$ Y. Sumino, K. H. Nagai, Y. Shitaka, D. Tanaka, K. Yoshikawa, H. Chate, and K. Oiwa, "Large-scale vortex lattice emerging from collectively moving microtubules," Nature 483, 448 (2012).

${ }^{52}$ A. Doostmohammadi, T. N. Shendruk, K. Thijssen, and J. M. Yeomans, "Onset of meso-scale turbulence in active nematics," Nat. Commun. 8, 15326 (2017).

${ }^{53}$ C. P. Brangwynne, G. H. Koenderink, F. C. MacKintosh, and D. A. Weitz, "Nonequilibrium microtubule fluctuations in a model cytoskeleton," Phys. Rev. Lett. 100, 118104 (2008).

${ }^{54}$ C. A. Weber, R. Suzuki, V. Schaller, I. S. Aranson, A. R. Bausch, and E. Frey, "Random bursts determine dynamics of active filaments," Proc. Natl. Acad. Sci. U. S. A. 112, 10703 (2015).

${ }^{55}$ S. C. Weber, A. J. Spakowitz, and J. A. Theriot, "Nonthermal ATPdependent fluctuations contribute to the in vivo motion of chromosomal loci," Proc. Natl. Acad. Sci. U. S. A. 109, 7338 (2012).

${ }^{56}$ A. Javer, Z. Long, E. Nugent, M. Grisi, K. Siriwatwetchakul, K. D. Dorfman, P. Cicuta, and M. Cosentino Lagomarsino, "Short-time movement of E. coli chromosomal loci depends on coordinate and subcellular localization," Nat. Commun. 4, 3003 (2013).

${ }^{57}$ A. Zidovska, D. A. Weitz, and T. J. Mitchison, "Micron-scale coherence in interphase chromatin dynamics," Proc. Natl. Acad. Sci. U. S. A. 110, 15555 (2013).

${ }^{58}$ I. Bronshtein, E. Kepten, I. Kanter, S. Berezin, M. Lindner, A. B. Redwood, S. Mai, S. Gonzalo, R. Foisner, Y. Shav-Tal, and Y. Garini, "Loss of lamin a function increases chromatin dynamics in the nuclear interior," Nat. Commun. 6, 8044 (2015).

${ }^{59}$ D. Osmanovic and Y. Rabin, "Dynamics of active Rouse chains," Soft Matter 13, 963 (2017).

${ }^{60} \mathrm{H}$. Vandebroek and C. Vanderzande, "Dynamics of a polymer in an active and viscoelastic bath," Phys. Rev. E 92, 060601 (2015). 
${ }^{61}$ B. Alberts, D. Bray, K. Hopkin, A. Johnson, J. Lewis, M. Raff, K. Roberts, and P. Walter, Essential Cell Biology (Garland Science, New York, 2013).

${ }^{62} \mathrm{~F}$. Kuttler and S. Mai, "Formation of non-random extrachromosomal elements during development, differentiation and oncogenesis," Semin. Cancer Biol. 17, 56 (2007).

${ }^{63}$ I. Cantat and C. Misbah, "Lift force and dynamical unbinding of adhering vesicles under shear flow," Phys. Rev. Lett. 83, 880 (1999).

${ }^{64} \mathrm{~A}$. Baumgaertner and R. Sambeth, "Polymerization-induced propulsion of a two-dimensional vesicle," J. Chem. Phys. 111, 5223 (1999).

${ }^{65} \mathrm{~S}$. Meßlinger, B. Schmidt, H. Noguchi, and G. Gompper, "Dynamical regimes and hydrodynamic lift of viscous vesicles under shear," Phys. Rev. E 80, 011901 (2009).

${ }^{66} \mathrm{M}$. K. Mitra, G. I. Menon, and R. Rajesh, "Thermodynamic behaviour of two-dimensional vesicles revisited," Eur. Phys. J. E 35, 30 (2012).

${ }^{67} \mathrm{C}$. Jeon, Y. Jung, and B.-Y. Ha, "A ring-polymer model shows how macromolecular crowding controls chromosome-arm organization in escherichia coli," Sci. Rep. 7, 11896 (2017).

${ }^{68}$ R. G. Winkler, P. Reineker, and L. Harnau, "Models and equilibrium properties of stiff molecular chains," J. Chem. Phys. 101, 8119 (1994).

${ }^{69}$ R. G. Winkler, "Deformation of semiflexible chains," J. Chem. Phys. 118, 2919 (2003).

${ }^{70}$ R. G. Winkler, "Semiflexible polymers in shear flow," Phys. Rev. Lett. 97, 128301 (2006).

${ }^{71}$ N. Samanta and R. Chakrabarti, "Chain reconfiguration in active noise," J. Phys. A: Math. Theor, 49, 195601 (2016).

${ }^{72}$ A. Kaiser, S. Babel, B. ten Hagen, C. von Ferber, and H. Löwen, "How does a flexible chain of active particles swell?," J. Chem. Phys. 142, 124905 (2015).

${ }^{73}$ T. Eisenstecken, A. Ghavami, A. Mair, G. Gompper, and R. G. Winkler, "Conformational and dynamical properties of semiflexible polymers in the presence of active noise," AIP Conf. Proc. 1871, 050001 (2017).

${ }^{74}$ S. Das, G. Gompper, and R. G. Winkler, "Confined active Brownian particles: Theoretical description of propulsion-induced accumulation," New J. Phys. 20, 015001 (2018).

${ }^{75} \mathrm{~B}$. Y. Ha and D. Thirumalai, "A mean-field model for semiflexible chains," J. Chem. Phys. 103, 9408 (1995).

${ }^{76} \mathrm{~A}$. Dua and B. J. Cherayil, "Effect of stiffness on the flow behavior of polymers," J. Chem. Phys. 113, 10776 (2000).
${ }^{77} \mathrm{~L}$. Harnau, R. G. Winkler, and P. Reineker, "Dynamic properties of molecular chains with variable stiffness," J. Chem. Phys. 102, 7750 (1995).

${ }^{78}$ M. D. El Alaoui Faris, D. Lacoste, J. Pécréaux, J. F. Joanny, J. Prost, and P. Bassereau, "Membrane tension lowering induced by protein activity," Phys. Rev. Lett. 102, 038102 (2009).

${ }^{79} \mathrm{M}$. Doi and S. F. Edwards, The Theory of Polymer Dynamics (Clarendon Press, Oxford, 1986).

${ }^{80} \mathrm{R}$. G. Winkler, "Diffusion and segmental dynamics of rodlike molecules by fluorescence correlation spectroscopy," J. Chem. Phys. 127, 054904 (2007).

${ }^{81}$ H. Risken, The Fokker-Planck Equation (Springer, Berlin, 1989).

${ }^{82} \mathrm{~J}$. Smrek and K. Kremer, "Small activity differences drive phase separation in active-passive polymer mixtures," Phys. Rev. Lett. 118, 098002 (2017).

${ }^{83}$ N. Ganai, S. Sengupta, and G. I. Menon, "Chromosome positioning from activity-based segregation," Nucleic Acids Res. 42, 4145 (2014).

${ }^{84}$ J. D. Halverson, J. Smrek, K. Kremer, and A. Y. Grosberg, "From a melt of rings to chromosome territories: The role of topological constraints in genome folding," Rep. Prog. Phys. 77, 022601 (2014).

${ }^{85}$ A. Goloborodko, J. F. Marko, and L. A. Mirny, "Chromosome compaction by active loop extrusion," Biophys. J. 110, 2162 (2016).

${ }^{86}$ A. Martín-Gómez, T. Eisenstecken, G. Gompper, and R. G. Winkler, "Active Brownian polymers with hydrodynamic interactions: Conformations and dynamics," Macromolecules (submitted).

${ }^{87}$ V. A. Martinez, J. Schwarz-Linek, M. Reufer, L. G. Wilson, A. N. Morozov, and W. C. K. Poon, "Flagellated bacterial motility in polymer solutions," Proc. Natl. Acad. Sci. U. S. A. 111, 17771 (2014).

${ }^{88}$ A. E. Patteson, A. Gopinath, M. Goulian, and P. E. Arratia, "Running and tumbling with E. coli in polymeric solutions," Sci. Rep. 5, 15761 (2015).

${ }^{89} \mathrm{~J}$. R. Gomez-Solano, A. Blokhuis, and C. Bechinger, "Dynamics of selfpropelled Janus particles in viscoelastic fluids," Phys. Rev. Lett. 116, 138301 (2016).

${ }^{90} \mathrm{~N}$. Narinder, C. Bechinger, and J. R. Gomez-Solano, "Memory-induced transition from a persistent random walk to circular motion for achiral microswimmers," Phys. Rev. Lett. 121, 078003 (2018). 